\title{
Evaluation of arterial stiffness in systolic heart failure
}

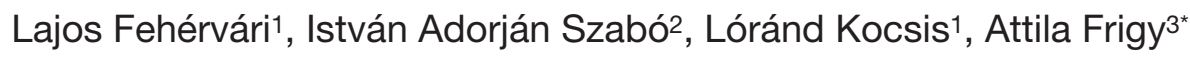 \\ 1. Department of Cardiology, Clinical County Hospital Mures, Targu Mures, Romania \\ 2. Department of Pathophysiology, George Emil Palade University of Medicine, Pharmacy, Science, and Technology of Targu Mures, \\ Romania \\ 3. Department of Internal Medicine IV, George Emil Palade University of Medicine, Pharmacy, Science, and Technology of Targu Mures, \\ Romania
}

\begin{abstract}
Objective: Micro- and macrovascular changes can occur in heart failure, and could influence its prognosis and management. In a prospective study, we proposed the evaluation of arterial stiffness (macrovascular function) and its correlations in patients with systolic heart failure. Methods: 40 patients (32 men, 8 women, mean age $63 \pm 2.9$ years), with hemodynamically stable systolic heart failure (left ventricular ejection fraction, $E F<40 \%$ ) were enrolled in the study. In every patient, beyond routine explorations (ECG, cardiac and carotid ultrasound, laboratory measurements), arterial stiffness was assessed by measuring pulse wave velocity (PWV). The correlations of PWV with clinical and echocardiographic characteristics were studied using t-test and chi-square test ( $p<0.05$ being considered for statistical significance). Results: The average PWV was $8.55 \pm 2.2 \mathrm{~m} / \mathrm{s}$, and 16 patients had increased PWV (>10 m/s). We found significantly higher PWV values in patients older than 65 years $(p<0.001)$, in patients with eGFR $<60 \mathrm{ml} / \mathrm{min} / 1.73 \mathrm{~m} 2(p<0.001)$, hypertension $(p=0.006)$, and increased $(>1 \mathrm{~mm})$ carotid intima-media thickness ( $p=0.016)$. PWV was found to be significantly lower when EF was $<30 \%$ ( $p=0.049)$. Furthermore, the presence of an increased PWV was correlated significantly with age ( $\mathrm{p}<0.001)$, and (with borderline significance) with eGFR $<60 \mathrm{ml} / \mathrm{min}^{\prime} / .73 \mathrm{~m}{ }^{2}$ and, inversely, with $\mathrm{EF}<30 \%$. Conclusions: Increased arterial stiffness reflected by high PWV is frequently present in patients with systolic heart failure, and is mainly correlated with general risk factors of arterial involvement. Low EF, due to low stroke volume and decreased systolic arterial wall tension can influence the values and the interpretation of PWV.
\end{abstract}

Keywords: systolic heart failure, arterial stiffness, pulse wave velocity

Received 7 November 2020 / Accepted 22 February 2021

\section{Introduction}

Heart failure (HF) is a result of cardiac structural and/or functional abnormalities, which determine low cardiac output and high filling pressures, responsible for clinical picture, consisted mainly of dyspnea, fatigue and signs of congestion. Systolic HF (HF with reduced ejection fraction - HFrEF) is associated with increased mortality and frequent hospital admissions. Its pathophysiology is complex, and involves central and peripheral mechanisms as well [1].

Arterial function, both its micro- and macrovascular component, has become a target for evaluation and therapy in many pathological conditions, including HF, vascular dysfunction having a well-known or putative role in cardiovascular disease mechanisms. Pulse wave velocity (PWV) has emerged in the last decades as a potent marker of arterial stiffness, a key element of macrovascular arterial dysfunction. PWV has proven to be a stable and reproductible parameter, and its increase has prognostic significance in many cardiovascular and non-cardiovascular conditions, like hypertension, coronary heart disease and chronic kidney disease $[2,3]$. Due to the commercially available, validated devices, the measurement of PWV has become reliable in the everyday practice [4].

The role of arterial stiffness in diastolic dysfunction is a well-studied phenomenon, however, there are only few data regarding the relationship of arterial stiffness and sys-

* Correspondence to: Attila Frigy

E-mail: afrigy68@gmail.com tolic dysfunction with heart failure ( $\mathrm{HFrEF})$. The existing studies found a lower pressure augmentation and a shorter ejection duration in this setting, however PWV was not different from controls in the majority of cases [5].

Based on these data, in our study, we evaluated the arterial stiffness, reflected by PWV, in patients with HFrEF. We choose this, less studied area of HF research, keeping in mind the potential value of evaluating macrovascular dysfunction for clinical and prognostic purposes in $\mathrm{HF}$ patients.

\section{Material and methods}

In a prospective study we enrolled 40 patients ( 8 female, 32 male, mean age $63 \pm 12,9$ years) with HFrEF, hospitalized with acute HF during 2017-2019 in the Cardiology Department of the Clinical County Hospital Mures, Targu Mures, Romania. The diagnosis of HF was established using standard criteria, including clinical, ECG, echocardiographic and biomarker (NT-proBNP) data. All the patients were in sinus rhythm, and were included in the study on voluntary basis, when they reached hemodynamic stability (blood pressure and heart rate in normal range, no signs of pulmonary or systemic congestion). In every patient, we recorded the most important general (e.g., sex, age) and clinical data (e.g., etiology of HF, underlying heart diseases) and the findings of the routine cardiac evaluation at inclusion, consisted of clinical examination, electrocardiography and echocardiography. Ischemic etiology was considered in the case of old myocardial infarction and/or significant stenoses on a previous coronarography. 
Left ventricular ejection fraction was determined using the modified biplane Simpson method [6]. The results of the last available routine laboratory tests were also noted. For evaluation of the macrovascular function we measured arterial stiffness, the ankle-brachial index (ABI) and the carotid intima-media thickness (IMT).

Arterial stiffness was evaluated using the Mobil-OGraph NG device (IEM GmbH, Stolberg, Germany) which uses a cuff-based (single point measurement - the dominant upper arm) oscillometric, non-invasive method for measuring PWV. The recorded pressure data were processed by the HMS Client Server Hypertension Management software (IEM GmbH, Stolberg, Germany), and aortic PWV was calculated. ABI and carotid IMT were measured according with current guidelines, using commercially available devices (peripheral CW Doppler device; $7 \mathrm{MHz}$ linear ultrasound probe - Aloka Prosound Alpha 10 machine) [ 7,8$]$.

The different characteristics of patient population are presented on Table I.

Table I. The main charactersitics of study population

\begin{tabular}{lc}
\hline Characteristic & Value ( \pm SD) \\
\hline Mean age (years) & $62.4 \pm 12.8$ \\
Female/Male (nr.) & $8 / 32$ \\
Ischemic etiology of heart failure (\%) & 34.1 \\
Diabetes (\%) & 19.5 \\
eGFR<60 ml/min/1.73 m2 (\%) & 24.4 \\
Hypertension (\%) & 46.3 \\
Mean PWV (m/s) & $8.55 \pm 2.2$ \\
PWV >10 m/s (\%) & 40 \\
Left ventricular ejection fraction (EF, \%) & 30.7 \\
EF<30\% (\%) & 48.8 \\
Carotid intima-media thickness >1 mm (\%) & 21.1 \\
Ankle-brachial index<0.9 (\%) & 22.0 \\
\hline eGFR: estimated glomerular filtration rate; PWV: pulse wave velocity; EF: ejection fraction
\end{tabular}

To find out the associations of increased arterial stiffness, reflected by increased PWV $(>10 \mathrm{~m} / \mathrm{s})$, [9] the general, clinical, echocardiographic and vascular variables of the patients were correlated with PWV values using bidirectional association analysis, by t-test and chi-square test ( $\mathrm{p}<0.05$ was considered statistically significant). Data processing was performed using the Microsoft Excel v14.0. and the IBM SPSS Statistics V22.0 software. Data handling respected all the time the Declaration of Helsinki, the patients signed a written informed consent, and the study had been approved (3865/01.03.2016) by the Ethical Committee of Clinical County Hospital Mures.

\section{Results}

The PWV values were found to be increased $(>10 \mathrm{~m} / \mathrm{s}$ ) in 16 patients (40\%), the average PWV being $8.55 \pm 2.2$ $\mathrm{m} / \mathrm{s}$. The bidirectional analysis of the relationship between the diverse patients' characterisitics and PWV values are presented on Table II and Table III. The PWV proved to be significantly higher in patients with age $>65$ years, eGFR $<60 \mathrm{ml} / \mathrm{min} / 1.73 \mathrm{~m}^{2}$ (chronic kidney disease, eGFR - estimated glomerular filtration rate - calculated using the Modification of Diet in Renal Disease, MDRD, formula), hypertension and increased carotid intima-media thickness, and significantly lower when left ventricular ejection fraction (EF) was $<30 \%$ (t-tests, see Table II). Furthermore, the presence of an increased PWV was significantly, positively, correlated with age, and (with borderline significance) with chronic kidney disease, and, inversely, with lover EF (chi-square tests, see Table III). As expected, pulse pressure, a classical clinical measure of arterial stiffness [2], was significantly higher in the group of patients with high PWV $(54.1 \pm 11.1 \mathrm{mmHg}$ vs. $44.3 \pm 10.3 \mathrm{mmHg}$,

Table II. The PWV values in function of patients' characteristics (t-test)

\begin{tabular}{|c|c|c|c|}
\hline Characteristics (cut-off criteria) & PWV value $($ mean \pm SD) & PWV value (mean $\pm S D)$ & p \\
\hline Age $(</>65$ years $)$ & $7.16 \pm 0.92$ & $10.85 \pm 1.33$ & $<0.001$ \\
\hline Gender (female/male) & $9.22 \pm 2.26$ & $8.85 \pm 2.18$ & 0.685 \\
\hline HF etiology (ischemic/non-ischemic) & $9.54 \pm 2.20$ & $8.97 \pm 2.14$ & 0.698 \\
\hline Diabetes $(\mathrm{Y} / \mathrm{N})$ & $9.40 \pm 2.22$ & $8.80 \pm 2.18$ & 0.508 \\
\hline eGFR $<60 \mathrm{ml} / \mathrm{min} / 1.73 \mathrm{~m} 2(\mathrm{Y} / \mathrm{N})$ & $10.86 \pm 1.63$ & $8.27 \pm 1.95$ & $<0.001$ \\
\hline Hypertension $(\mathrm{Y} / \mathrm{N})$ & $9.89 \pm 2.05$ & $8.03 \pm 1.93$ & 0.006 \\
\hline$E F<30 \%(Y / N)$ & $8.21 \pm 1.99$ & $9.56 \pm 2.17$ & 0.049 \\
\hline Carotid intima-media thickness $>1 \mathrm{~mm}(\mathrm{Y} / \mathrm{N})$ & $10.58 \pm 1.43$ & $8.51 \pm 2.18$ & 0.016 \\
\hline Ankle-brachial index<0,9 (Y/N) & $8.91 \pm 2.48$ & $8.92 \pm 2.12$ & 0.989 \\
\hline
\end{tabular}

Y:yes (present); N: no (not present)

Table III. Correlations of PWV $>10 \mathrm{~m} / \mathrm{s}$ (chi-square test)

\begin{tabular}{|c|c|c|}
\hline Characteristics (cut-off criteria) & $\%$ of patients with $P W V>10 \mathrm{~m} / \mathrm{s}$ & p \\
\hline Age $(</>65$ years $)$ & $0 / 73.7$ & $<0.001$ \\
\hline Gender (female/male) & $57.1 / 30.3$ & 0.186 \\
\hline Etiology (ischemic/non-ischemic) & $35.7 / 37.1$ & 0.434 \\
\hline Diabetes $(\mathrm{Y} / \mathrm{N})$ & $37.5 / 34.4$ & 0.869 \\
\hline eGFR <60 ml/min/1.73 m2 (Y/N) & $60.0 / 26.7$ & 0.06 \\
\hline Hypertension (Y/N) & $47.4 / 23.8$ & 0.117 \\
\hline$E F<30 \%(Y / N)$ & $21.1 / 47.6$ & 0.075 \\
\hline Carotid intima-media thickness $>1 \mathrm{~mm}(\mathrm{Y} / \mathrm{N})$ & $50.0 / 31.0$ & 0.328 \\
\hline Ankle-brachial index <0,9 (Y/N) & $33.3 / 35.5$ & 0.905 \\
\hline
\end{tabular}


$\mathrm{p}=0.007)$, due to the higher values of systolic blood pressure $(129.2 \pm 14.3 \mathrm{mmHg}$ vs. $116.0 \pm 13.1, \mathrm{p}=0.005)$.

\section{Discussion}

For methodological and clinical reasons we studied the prevalence and correlations of increased arterial stiffness (reflected by elevated PWV) in patients with systolic heart failure.

Increased arterial stiffness represents a major macrovascular involvement, with well-known consequences on central hemodynamics: increased central systolic blood pressure, increased left ventricular afterload and decreased diastolic coronary supply. These consequences are the results of increased PWV, which causes an early arrival (during systole instead of diastole) of the reflected pulse wave. All of the above factors could influence left ventricular performance, contributing to the aggravation/progression of heart failure, independently of left ventricular ejection fraction $[10,11]$.

The development of increased arterial stiffness is related to arteriosclerosis, a process which is driven by endothelial dysfunction (ED) and vascular ageing [12]. In systolic HF, due to a complex pathophysiological mechanism (e.g., decreased arterial wall tension, neurohormonal activation) there is a fall in endothelial nitric oxide (NO) synthase expression, with low endothelium-derived NO production. This endothelial dysfunction has been documented both in the peripheral and coronary arteries in patients with HFrEF [13]. Furthermore, the degree of endothelial dysfunction can be used as a marker of long-term prognosis in these patients. It has been demonstrated that radial and brachial artery post-ischemic vasodilation is inversely correlated with mortality in patients with HF [14].

In our patient population the prevalence of increased arterial stiffness was high (40\%), however, due to the inverse relationship of EF with PWV, revealed by our study, this proportion could be even higher. Thus, macrovascular dysfunction seems to be an important co-factor of altered left ventricular hemodynamics in patients with HFrEF. On the other hand, increased arterial stiffness in HFrEF patients was correlated with the same risk factors as in the general population, including age, hypertension and chronic kidney disease [15]. Similarly, we found a good correlation of increased arterial stiffness with subclinical carotid disease $[16,17]$.

Our study has a few limitations, including the relatively small sample size, which could serve as an explanation for the lack of correlations of arterial stiffness with the presence of diabetes and ischemic heart disease. Also, we do not performed the study of correlations of arterial stiffness with natriuretic peptides and medical treatment. Despite the fact that the patients enrolled in the study were hospitalized, already stabilized patients, medical treatment and natriuretic peptide values cannot be considered constant and representative in this setting. Our study, a pilot research, could serve as starting point for a more complex evaluation of the relationship between arterial stiffness and systolic HF with potential therapeutic and prognostic yield. For instance, the prognostic value of PWV in systolic HF is still controversial, as data derived from the Eplerenone Post-Acute Myocardial Infarction Heart Failure Efficacy and Survival Study (EPHESUS) underscores its importance, while other studies did not find it useful in prognostic stratification $[18,19]$.

\section{Conclusions}

Increased arterial stiffness as an expression of macrovascular arterial dysfunction is frequently present in patients with systolic HF (HFrEF), being a sign and consequence of altered endothelial function and vascular ageing. In our patient population, increased stiffness was found to be correlated with the general risk factors of arterial involvement. Low EF, due to low stroke volume and decreased systolic arterial wall tension can influence the value of PWV and its interpretation. Concerning the patients with low EF and high PWW, a recommendation of a more closed monitoring could be rised, as their prognosis can be worse due to a higher load. Therapeutic implications can be considered too: the possible, beneficial effect of vasodilators in patients with high PWV. These and other questions certainly warrant further research of the field.

\section{Authors' contribution}

LF (conception and design; acquisition of data; analysis and interpretation of data; drafting the article; revising critically the article; final approval)

$\mathrm{IAS}_{z}$ (acquisition of data; analysis and interpretation of data; final approval)

LK (analysis and interpretation of data, revising critically the article; final approval)

AF (conception and design; acquisition of data; analysis and interpretation of data; drafting the article; revising critically the article; final approval)

\section{Acknowledgement}

The research was founded by the Research Grant with private financing - nr.524/17.01.2017 of the G.E. Palade University of Medicine, Pharmacy, Science and Technology of Tirgu Mures

\section{Conflict of interest}

None to declare.

\section{Informed consent statement}

Data handling respected all the time the Declaration of Helsinki, the patients signed a written informed consent, and the study had been approved (3865/01.03.2016) by the Ethical Committee of Clinical County Hospital Mures.

\section{References}

1. Ponikowski P, Voors AA, Anker SD, et al. 2016 ESC Guidelines for the diagnosis and treatment of acute and chronic heart failure: the Task Force for the diagnosis and treatment of acute and chronic heart failure 
of the European Society of Cardiology (ESC) developed with the special contribution of the Heart Failure Association (HFA) of the ESC. Eur Heart J. 2016;37:2129-2200. doi: 10.1093/eurhearti/ehw128.

2. Townsend RR. Arterial stiffness: recommendations and standardization. Pulse (Basel). 2017;4(Suppl 1):3-7. doi: 10.1159/000448454.

3. Dumor K, Shoemaker-Moyle M, Nistala R, Whaley-Connell A. Arterial stiffness in hypertension: an update. Curr Hypertens Rep. 2018;20:72. doi: 10.1007/s11906-018-0867-x.

4. Milan A, Zocaro G, Leone D, et al. Current assessment of pulse wave velocity: comprehensive review of validation studies. J Hypertens. 2019;37:1547-1557. doi: 10.1097/HJH.0000000000002081.

5. Weber T. Systolic and diastolic function as related to arterial stiffness. Artery Research 4:122-127. doi: 10.1016/j.artres.2010.10.033

6. Lang RM, Badano LP, Mor-Avi V, et al. Recommendations for cardiac chamber quantification by echocardiography in adults: an update from the American Society of Echocardiography and the European Association of Cardiovascular Imaging. J Am Soc Echocardiogr. 2015; 28: 1-39.e14.

7. Thurston B, Dawson J. Ankle Brachial Pressure Index: An update for the vascular specialist and general practitioner. Vascular. 2019;27:560-570. doi: 10.1177/1708538119842395.

8. Bots ML, Evans GW, Tegeler CH, Meijer R. Carotid intima-media thickness measurements: relations with atherosclerosis, risk of cardiovascular disease and application in randomized controlled trials. Chin Med J (Engl). 2016;129:215-26. doi: 10.4103/0366-6999.173500.

9. Williams B, Mancia G, Spiering W, et al. 2018 ESC/ESH Guidelines for the management of arterial hypertension. Eur Heart J. 2018 1;39:30213104. doi: 10.1093/eurheartj/ehy339.

10. Palombo C, Kozakova M. Arterial stiffness, atherosclerosis and cardiovascular risk: Pathophysiologic mechanisms and emerging clinical indications. Vascul Pharmacol. 2016;77:1-7. doi: 10.1016/j. vph.2015.11.083.

11. Sequí-Domínguez I, Cavero-Redondo I, Álvarez-Bueno C, Pozuelo-
Carrascosa DP, Nuñez de Arenas-Arroyo S, Martínez-Vizcaíno V. Accuracy of pulse wave velocity predicting cardiovascular and allcause mortality. A systematic review and meta-analysis. J Clin Med. 2020;9:2080. doi: 10.3390/jcm9072080.

12. Wang M, Monticone RE, McGraw KR. Proinflammation, profibrosis, and arterial aging. Aging Med (Milton). 2020;3:159-168. doi: 10.1002/ agm2.12099.

13. Smith CJ, Sun D, Hoegler C, et al. Reduced gene expression of vascular endothelial NO synthase and cyclooxygenase- 1 in heart failure. Circ Res. 1996;78: 58-64.

14. Katz SD, Hryniewicz K, Hriljac I, et al. Vascular endothelial dysfunction and mortality risk in patients with chronic heart failure. Circulation. 2005; 111:310-314.

15. Feola M, Testa M, Ferreri C, Rosso G, Rossi A, Ruocco G. The analysis of arterial stiffness in heart failure patients in comparison with healthy subjects and patients with cardiovascular risk factors. J Clin Med. 2019;8:1721. doi: 10.3390/jcm8101721.

16. Chirinos JA, Segers P, Hughes T, Townsend R. Large-artery stiffness in health and disease: JACC State-of-the-Art Review. J Am Coll Cardiol. 2019;74:1237-1263. doi: 10.1016/j.jacc.2019.07.012.

17. Singam NSV, Fine C, Fleg JL. Cardiac changes associated with vascular aging. Clin Cardiol. 2020;43:92-98. doi: 10.1002/clc.23313.

18. Regnault $\mathrm{V}$, Lagrange $\mathrm{J}$, Pizard $\mathrm{A}$, et al. Opposite predictive value of pulse pressure and aortic pulse wave velocity on heart failure with reduced left ventricular ejection fraction: insights from an Eplerenone Post-Acute Myocardial Infarction Heart Failure Efficacy and Survival Study (EPHESUS) substudy. Hypertension. 2014;63(1):105-111. doi: 10.1161/HYPERTENSIONAHA.113.02046

19. Dohaei A, Taghavi S, Amin A, Rahimi S, Naderi N. Does aortic pulse wave velocity have any prognostic significance in advanced heart failure patients? J Cardiovasc Thorac Res. 2017;9(1):35-40. doi: 10.15171/ jcvtr.2017.05 